\title{
Etude, par mesures ultrasonores, des premiers mouvements des lignes de dislocations dans l'aluminium pur, à basse température
}

\author{
$\mathrm{Ph}$. Deterre, P. Peguin et F. Vanoni \\ Centre d'Etudes Nucléaires de Grenoble, Département de Recherche Fondamentale, Section de Physique du Solide, \\ 85X, 38041 Grenoble Cedex, France
}

(Reçu le 23 février 1979, révisé le 6 juin 1979, accepté le 11 juin 1979)

\begin{abstract}
Résumé. - Sur de l'aluminium de haute pureté nous avons réalisé des mesures d'atténuation d'ondes ultrasonores au cours de l'application d'une contrainte mécanique. Nous avons ainsi étudié le mouvement des lignes de dislocation à basse température. Trois phénomènes ont été mis en évidence en fonction de la contrainte mécanique croissante :- Le raidissement, dès l'application de très faibles contraintes, on observe dans certaines conditions, une diminution de l'atténuation et une augmentation de vitesse. - L'amollissement, à contraintes plus élevées et à des températures supérieures à $15 \mathrm{~K}$ on observe l'inversion des variations des deux paramètres ultrasonores. - La microplasticité, à partir d'un certain seuil de contrainte, on peut caractériser le tout début de la déformation plastique. L'interprétation montre que les deux premiers phénomènes qui sont réversibles et reproductibles, peuvent être reliés à l'existence de l'énergie de Peierls dans les métaux c.f.c.
\end{abstract}

\begin{abstract}
Ultrasonic measurements have been performed during application of a load stress, on very aluminium at low temperatures. The variations of the anelastic characteristics were attributed to the movements of dislocations. Versus the increasing applied stress three phenomena were observed. - The stiffening which appears reversibly at low applied stresses. - The softening, at higher stresses the ultrasonic attenuation increases while modulus decreases reversibly. - At last, the microplasticity characterizing the binginning of the plastic deformation has been characterized. The interpretation shows that stiffening and softening can be related to the existence of the Peierls-Nabarro energy in the c.f.c. metals.
\end{abstract}

Introduction. - L'étude de la présence et des mouvements des dislocations dans les solides cristallins a beaucoup progressé ces dernières années, en particulier, grâce à la complémentarité des techniques utilisées : les essais mécaniques ont permis d'approfondir l'interprétation des phénomènes de plasticité, tandis que les expériences de frottement interne contribuaient largement à la connaissance des mécanismes anélastiques. Il reste quelques incertitudes sur les processus physiques contrôlant les premiers mouvements irréversibles des dislocations dans les métaux c.f.c. à basse température; entre autres, le rôle de l'énergie de Peierls est encore mal déterminé $[1,2,3]$. Pour explorer ces domaines anélastique et microplastique, nous avons utilisé une technique qui consiste à mettre en mouvement les dislocations par application d'une contrainte mécanique et à étudier leur mobilité à l'aide d'ondes ultrasonores de faible niveau [4]. Cette technique a été adaptée aux faibles contraintes [5] et nous l'avons étendue au domaine des basses températures.

Nous présentons dans cet article les résultats obtenus dans l'aluminium de haute pureté, puis nous les discuterons sur la base du modèle du potentiel de Peierls-Nabarro, en montrant qu'il est capable de rendre compte de nos observations.

1. Technique expérimentale. - La méthode adoptée consiste à appliquer une contrainte par compression mécanique à un échantillon parallélépipédique $(10 \times 10 \times 15 \mathrm{~mm})$ et à enregistrer simultanément les variations d'atténuation et de vitesse d'une onde ultrasonore générée au sein du matériau. La contrainte, appliquée à vitesse lente $\left(\ell=10^{-6} \mathrm{~s}^{-1}\right)$ est considérée comme quasi statique. Le transducteur ultrasonore est un quartz collé sur une face latérale de l'échantillon à qui il transmet des ondes longitudinales de fréquence $10 \mathrm{MHz}$.

La génération du train d'attaque et les mesures ultrasonores sont assurées par un appareil original appelé échomètre [7,8]. Les détails techniques et les différents essais de l'appareillage sont décrits par ailleurs [6]. 
2. Matériau utilisé. - Les expériences ont été réalisées sur deux qualités d'aluminium :

a) Aluminium de pureté $99,999 \%$ (noté $5 \mathrm{~N}$ ) polycristallin. Après polissage les échantillons sont recuits $12 \mathrm{~h}$ à $700 \mathrm{~K}$ sous atmosphère d'hélium; le diamètre des grains est d'environ $1 \mathrm{~mm}$.

b) Aluminium de pureté 99,9999\% (noté 6N) monocristallin, élaboré par la méthode de zone fondue par le C.N.R.S. de Vitry/Seine.

Après polissage les échantillons sont recuits $10 \mathrm{~h}$ à $600 \mathrm{~K}$ sous atmosphère d'hélium. L'axe de compression est orienté selon $\langle 03 \overline{2}\rangle$; quatre systèmes de glissement peuvent être sollicités par une contrainte dirigée selon cette direction, les facteurs de Schmid $S$ [9] correspondants sont portés au tableau I. Les

Tableau I.

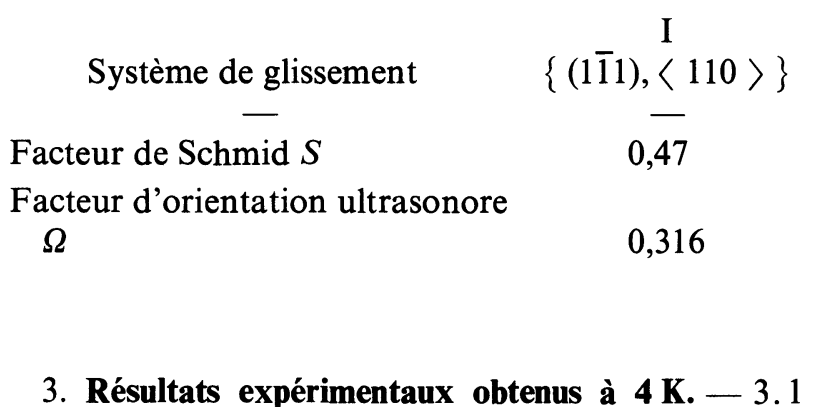
ALluRE GÉNÉRALE DES COURBES EXPÉRIMENTALES. Les échantillons sont déformés à $4 \mathrm{~K}$ par cycles de contrainte d'amplitude croissante et variant de 0 à $+\sigma$, au cours de cette déformation les variations d'atténuation $\Delta \alpha$ et de vitesse relative $\Delta V / V$ ultrasonores sont enregistrées en fonction de la contrainte quasi statique $\sigma$. Les figures 1 et 2 représentent les courbes expérimentales obtenues sur les deux qualités d'aluminium $5 \mathrm{~N}$ et $6 \mathrm{~N}$. On distingue nettement aux faibles contraintes, un premier mode de variation des paramètres ultrasoniques puis, pour $\sigma$ plus important une croissance rapide de l'atténuation accompagnée d'une baisse importante de la vitesse relative, qu'on associera à la microplasticité.

3.1.1 Le raidissement. - Intéressons-nous tout d'abord à la première partie des courbes qui correspond à des valeurs de la contrainte inférieures à la contrainte maximale atteinte au cycle précédent. Nous avons vérifié que dans ce domaine les variations de la déformation et des paramètres ultrasonores sont réversibles. Nous remarquons, dans le cas de l'aluminium $6 \mathrm{~N}$ un phénomène rarement observé : l'atténuation diminue et la vitesse augmente pour des contraintes croissantes. Pour caractériser le comportement de l'échantillon nous mesurons les pentes, notées $p_{\alpha}$ et $p_{v}$, des courbes $\Delta \alpha=f(\sigma)$ et $\Delta V / V=f(\sigma)$ au voisinage de l'origine pour $\sigma$ croissant. Dès la première montée de contrainte dans $\mathrm{Al} 6 \mathrm{~N}$ et après une déformation suffisante dans $\mathrm{Al} 5 \mathrm{~N}, p_{\alpha}$ est négatif. Cette décroissance de l'atténuation ultrasonore sous ondes ultrasonores se propagent selon la direction $\langle 14.6 .9\rangle$ qui a, par rapport aux systèmes de glissement, des facteurs d'orientation ultrasonores $\Omega$ [10] également portés au tableau I. Le facteur d'orientation, qui est égal au rapport de l'énergie élastique emmagasinée dans le système de glissement considéré, sur l'énergie élastique totale, traduit l'efficacité des dislocations appartenant à ce système pour affecter l'onde ultrasonore.

Les déformations imposées à l'échantillon sont inférieures à $0,3 \%$ et ne dépassent pas le stade I [9]. Les systèmes de glissement notés III et IV ne sont pas activés. Le facteur $\Omega$ du système II est très faible, nous considèrerons donc que nos mesures ultrasonores ne sont sensibles qu'aux mouvements des dislocations du système $I$. l'effet d'une contrainte appliquée avait été prévue par Suzuki et Elbaum [11], puis par G. Alefeld [12] ; elle a été observée par Vincent et Perez [13] dans l'aluminium, par Pinatti et Roberts [14] dans le tungstène. Il lui correspond une augmentation de vitesse relative, donc de module. Il faut toutefois noter que cette augmentation de vitesse est modérée par l'existence des constantes élastiques du $3^{\mathrm{e}}$ ordre, liées aux effets anharmoniques du réseau et qui conduisent à une baisse de vitesse de l'ordre de $1,5 \times 10^{-11} \mathrm{~Pa}^{-1}$ (comme nous l'avons estimé à partir d'un essai effectué sur une éprouvette de duraluminium, matériau dans lequel on peut considérer que les dislocations ne sont pas mobiles); cette estimation est d'ailleurs sensiblement en accord avec le résultat tiré de l'analyse théorique de Thurston et Brugger [15].

$\mathrm{La}$ comparaison des figures 1 et 2 montre que ce phénomène (diminution d'atténuation et augmentation de vitesse) est particulièrement net dans le cas de l'aluminium $6 \mathrm{~N}$. Il est moins apparent dans le cas de l'aluminium $5 \mathrm{~N}$ car le rôle des impuretés, pouvant conduire à des effets de désancrage [16], est important; l'effet anharmonique suffit alors pour compenser largement l'augmentation de vitesse.

On a choisi de qualifier cet effet de raidissement pour le distinguer de durcissement qu'on associe habituellement à l'augmentation de limite élastique.

3.1.2 La microplasticité. - Aux contraintes plus élevées on note une variation importante et irréversible des paramètres ultrasonores : augmentation 

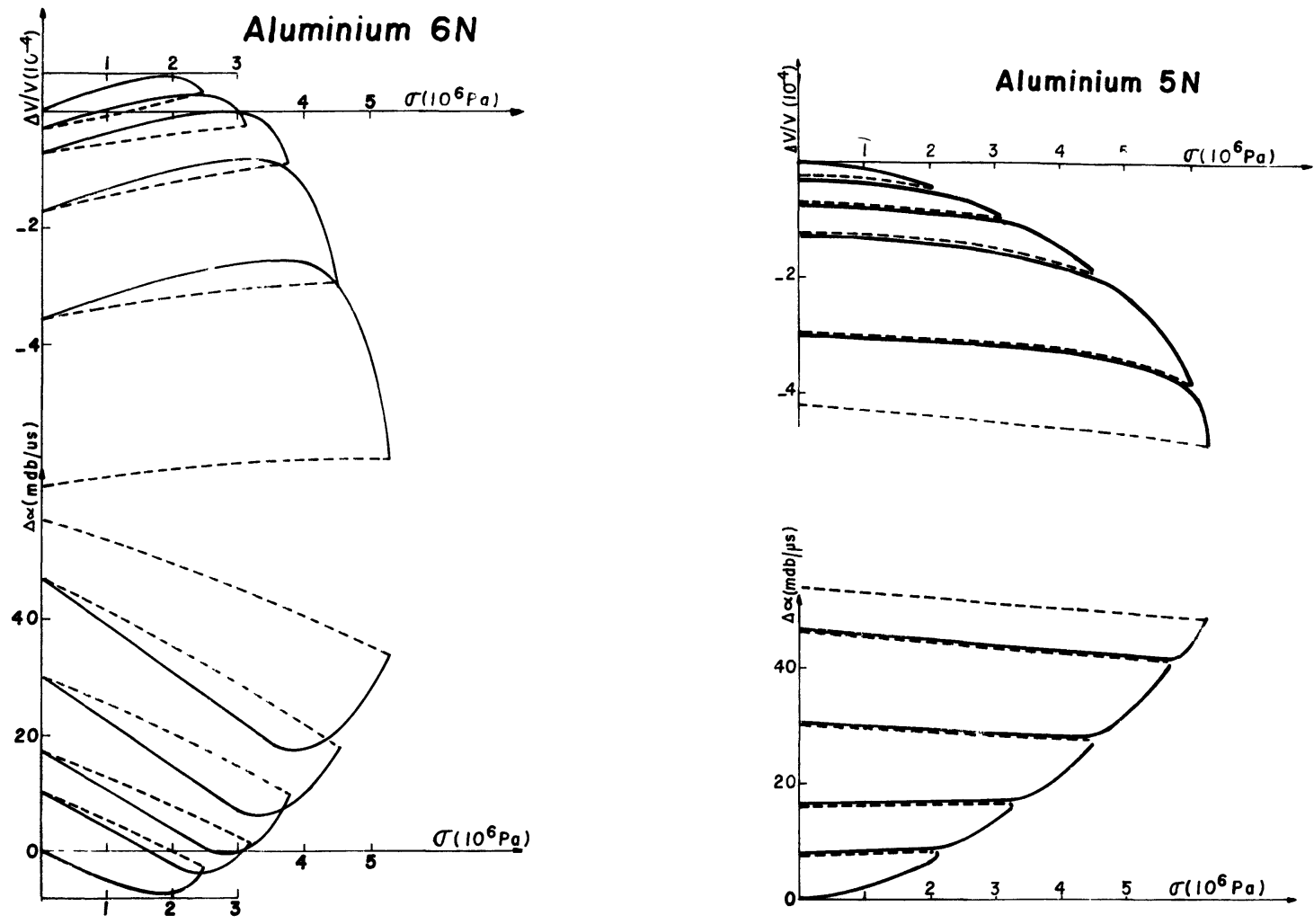

Fig. 1 et 2. - Variations de l'atténuation $\Delta \alpha$ et de vitesse relative $\Delta V / V$ ultrasonores et fonction de la contrainte $\sigma$ (cycles croissants). Température de l'essai : $4 \mathrm{~K}$.

[Changes of attenuation $\Delta \alpha$ and relative velocity $\Delta V / V$ versus quasistatic bias stress $\sigma$. Temperature test : $4 \mathrm{~K}$.]

de l'atténuation et diminution de la vitesse ultrasonore, caractéristiques de la déformation plastique $\mathrm{du}$ matériau [4]. Nous définissons la contrainte de microplasticité $\sigma_{\mathrm{m}}$ comme étant celle qui est nécessaire pour induire une diminution irréversible de vitesse relative ultrasonore égale à $2,5 \times 10^{-5}$. Nous repérons ainsi avec précision le début de la plasticité (microplasticité) puisque $\sigma_{\mathrm{m}}$ correspond à une déformation plastique de l'ordre de $10^{-6}$.

\subsection{ETUde DU RAIDISSEMENT OBSERVÉ A $4 \mathrm{~K}$. -} $p_{v}$ et $p_{\alpha}$ caractérisent le raidissement, étudions leurs variations en fonction de différents paramètres.

La figure 3 représente les variations de $p_{\mathrm{v}}$ et de $p_{\alpha}$ en fonction de la contrainte maximale atteinte au cycle précédent $\sigma_{\mathrm{e}}$ (contrainte d'écrouissage) le raidissement est globalement croissant avec l'écrouissage du matériau.

Des recuits de $15 \mathrm{mn}$ à différentes températures ont été opérés sur un échantillon d'aluminium $6 \mathrm{~N}$ déformé de $0,1 \%$ à $4 \mathrm{~K}$. Après chaque recuit, l'échantillon est de nouveau soumis, à $4 \mathrm{~K}$, à une contrainte statique inférieure à celle ayant produit la déformation initiale de $0,1 \%$. Les pentes $p_{\mathrm{v}}$ et $p_{\alpha}$, ainsi mesurées décroissent en fonction de la température de recuit (voir Fig. 4). Après recuit à $80 \mathrm{~K}, p_{\alpha}$ est même positif.
4. Résultats obtenus à différentes températures d'essai. - Des essais à températures comprises entre $4 \mathrm{~K}$ et $150 \mathrm{~K}$ ont été réalisés sur un échantillon d'aluminium $6 \mathrm{~N}$ où un état de référence a été établi par une déformation de $0,1 \%$ suivie d'un recuit de $2 \mathrm{H}$ à $210 \mathrm{~K}$ (il a été démontré, par ailleurs [5, 17] que cet état correspond à une mobilité maximale des dislocations). Nous avons reporté (Fig. 5) les courbes

$$
\Delta \alpha=f(\sigma) \text { et } \quad \Delta V / V=f(\sigma)
$$

pour $\sigma<\sigma_{\mathrm{m}}$ obtenues à différentes températures d'essai. En outre, nous avons reporté aux figures 6 et 7, les mesures de $\sigma_{\mathrm{m}}, p_{\alpha}$ et $p_{\mathrm{v}}$ en fonction de la température d'essai.

Les courbes de la figure 5 révèlent, qu'à $15 \mathrm{~K}$ et audessus, l'effet de raidissement est suivi, à partir d'une contrainte comprise entre 75 et $100 \times 10^{4} \mathrm{~Pa}$ d'une augmentation d'atténuation corrélée à une diminution de vitesse relative, toutes deux réversibles au cours du cycle de contrainte (les courbes de variation de $\Delta V / V$ et $\Delta \alpha$ pour $\sigma$ croissant et décroissant sont quasiment superposables). Nous avons appelé ce nouveau phénomène amollissement préféré au mot d'adoucissement qui décrit plutôt une baisse de la contrainte d'écoulement plastique [18, 19]. 


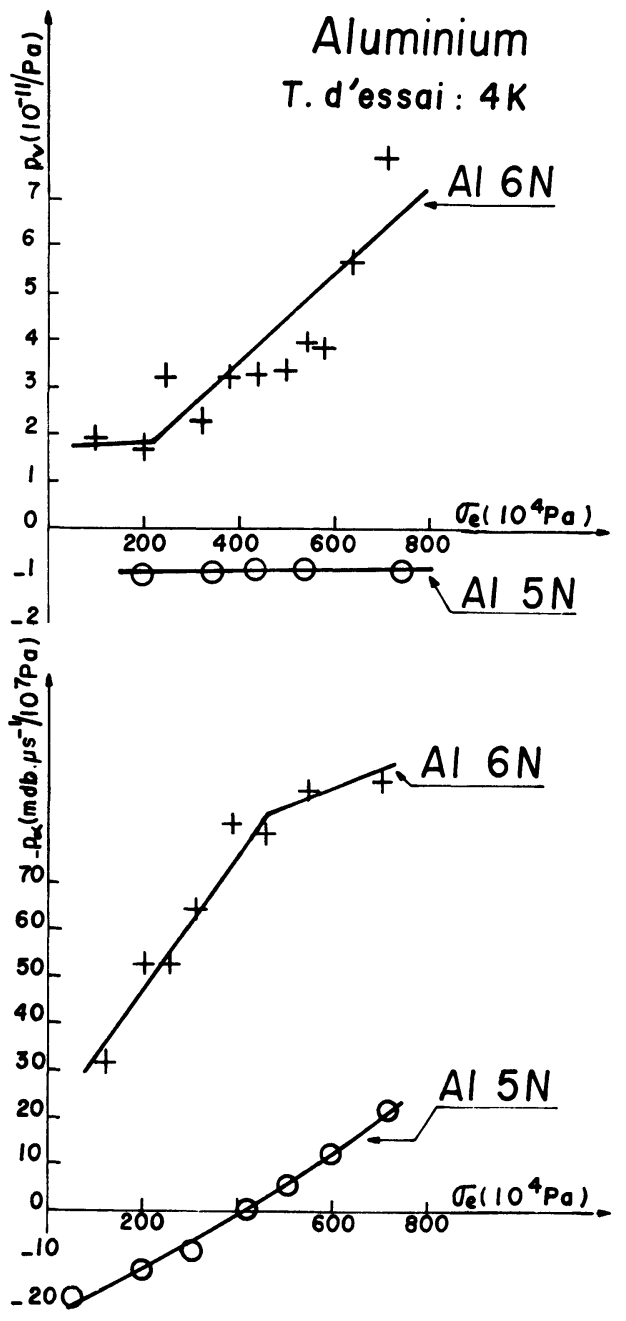

Fig. 3. - Variations de $p_{\mathrm{v}}$ et $-p_{\alpha}$ en fonction de la contrainte d'écrouissage $\sigma_{\mathrm{e}}$ pour les deux qualités d'aluminium $6 \mathrm{~N}$ et $5 \mathrm{~N}$.

[Changes of slopes $p_{\mathrm{v}}$ and $-p_{\alpha}$ versus cold-worked stress $\sigma_{\mathrm{e}}$ for two aluminium of purity $6 \mathrm{~N}$ and $5 \mathrm{~N}$.]

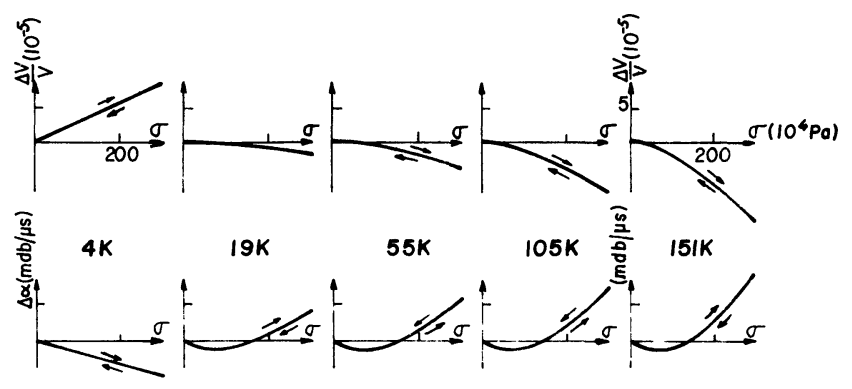

Fig. 5. - Variation de l'atténuation $\Delta \alpha$ et de la vitesse relative $\Delta V / V$ en fonction de la contrainte $\sigma$ dans l'aluminium $6 \mathrm{~N}$ écroui de $0,1 \%$ à $4 \mathrm{~K}$ et recuit $2 \mathrm{~h}$ à $210 \mathrm{~K}$ à différentes températures d'essai.

[Changes of attenuation $\Delta \alpha$ and relative velocity $\Delta V / V$ versus bias stress $\sigma$ in $6 \mathrm{~N}$ aluminium cold-worked of $0.1 \%$ at $4 \mathrm{~K}$ and annealed 2 hours at $210 \mathrm{~K}$, for several temperatures tests.]

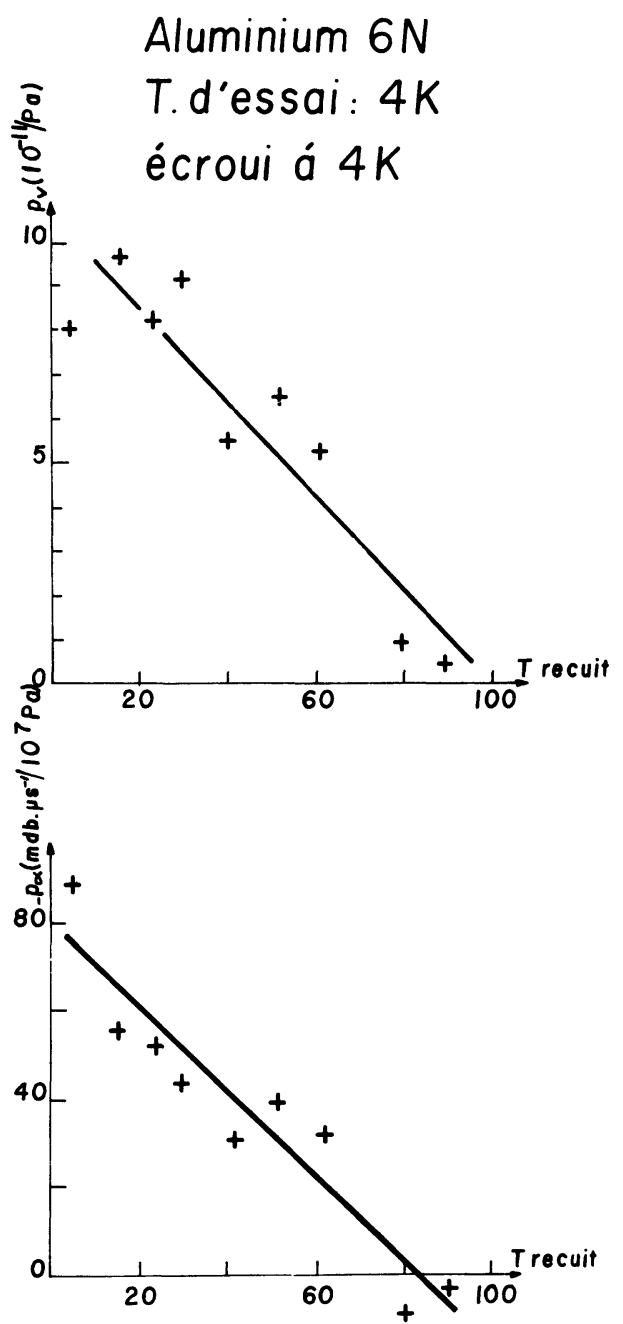

Fig. 4. - Variation des pentes $p_{\mathrm{v}}$ et $-p_{\alpha}$ à $4 \mathrm{~K}$ en fonction de la température de recuit isochrone $(15 \mathrm{~min})$ dans l'aluminium $6 \mathrm{~N}$ écroui de $0,1 \%$ à $4 \mathrm{~K}$.

[Changes of slopes $p_{\mathrm{v}}$ and $-p_{\alpha}$ at $4 \mathrm{~K}$ versus the isochronal annealing temperature in aluminium $6 \mathrm{~N}$ cold-worked of $0.1 \%$ at $4 \mathrm{~K}$.]

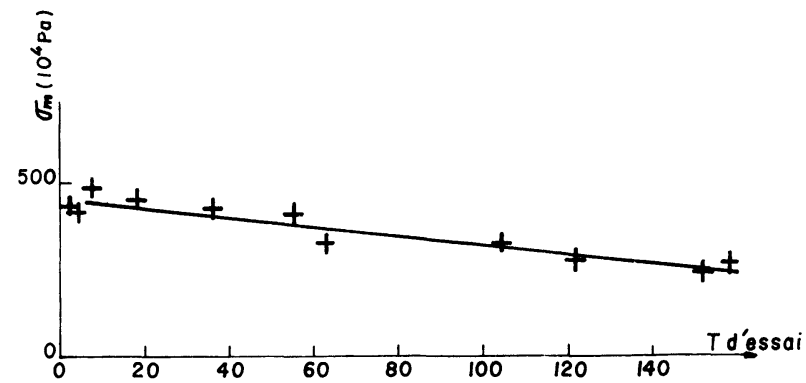

Fig. 6. - Variation de la contrainte de microplasticité $\sigma_{\mathrm{m}}$ en fonction de la température d'essai, dans l'aluminium $6 \mathrm{~N}$.

[Changes of microplasticity stress $\sigma_{\mathrm{m}}$ versus the temperature test in $6 \mathrm{~N}$ aluminium.] 

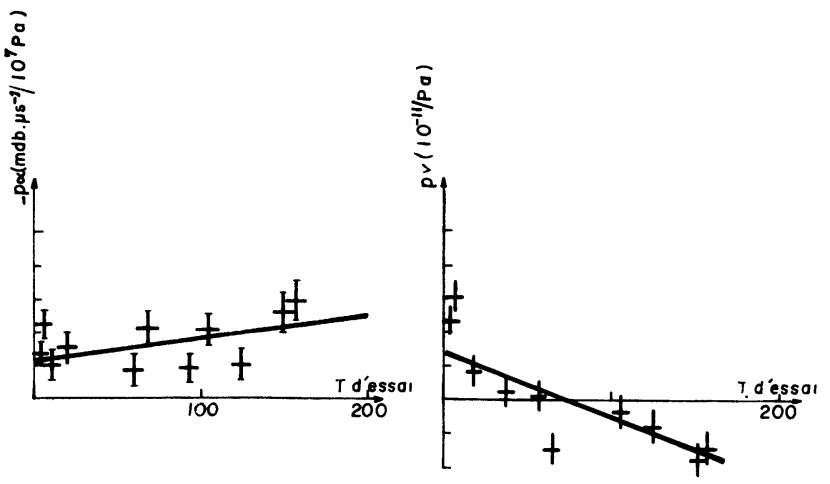

Fig. 7. - Variation des pentes $-p_{\alpha}$ et $p_{\mathrm{v}}$ en fonction de la température d'essai dans l'aluminium $6 \mathrm{~N}$.

[Changes of slopes $p_{\mathrm{v}}$ and $-p_{\alpha}$ versus the temperature test in $6 \mathrm{~N}$ aluminium.]
5. Discussion. - 5.1 INTERPRÉTATION DU RAIDISSEMENT. - Le fait que le raidissement soit nettement favorisé par une meilleure pureté du métal (Figs. 2 et 3) nous amène à interpréter ce phénomène par un mécanisme intrinsèque lié aux mouvements des dislocations. Un tel effet ne peut pas être expliqué à partir $\mathrm{du}$ modèle de la corde vibrante mais serait associé à l'existence de contraintes de Peierls s'opposant aux mouvements des dislocations $[12,13]$. Une dislocation non parallèle aux directions de Peieris (direction dense) est formée d'une série de décrochements géométriques. Sous l'effet de la contrainte ces décrochements se déplacent vers une extrémité de la dislocation et s'empilent. Leur mobilité est alors fortement réduite et leur aptitude à atténuer l'onde ultrasonore diminue. La diminution d'atténuation et l'augmentation de module sont linéaires avec la contrainte appliquée [13] ; les caractéristiques $p_{\alpha}$ et $p_{\mathrm{v}}$ peuvent alors s'écrire

$$
\begin{aligned}
& p_{\alpha}=\frac{\partial \alpha}{\partial \sigma}=-5,44\left(\frac{\mu b^{2}}{E_{\mathrm{L}}}\right)^{2} \frac{1}{\Phi_{\max }} \Lambda L^{3}\left(\frac{S \Omega}{\mu}\right) \frac{\omega^{2} \tau\left[1-\left(\frac{\omega}{\omega^{0}}\right)^{2}\right]^{1 / 2}}{\left[1+(\omega \tau)^{2}+\left(\frac{\omega}{\omega^{0}}\right)^{4}-2\left(\frac{\omega}{\omega^{0}}\right)^{2}\right]^{3 / 2}} \\
& p_{\mathrm{v}}=\frac{\partial\left(\frac{\Delta V}{V}\right)}{\partial \sigma}=2,1 \times 10^{5}\left(\frac{\mu b^{2}}{E_{\mathrm{L}}}\right)^{2} \frac{1}{\Phi_{\max }} \Lambda L^{3} \frac{1-(\omega \tau)^{2}}{\left[1+(\omega \tau)^{2}\right]^{3 / 2}}\left(\frac{S \Omega}{\mu}\right) \text { pour } \omega \ll \omega_{0}
\end{aligned}
$$

avec

$$
\tau=\frac{B L^{2}}{\pi^{2} E_{\mathrm{L}} \sin \varphi} \quad \omega_{0}^{2}=\frac{E_{\mathrm{L}} \pi^{2} a \sin \varphi}{M_{\mathrm{K}} L^{2}}
$$

où $\omega:$ pulsation ultrasonore, $\omega_{0}:$ pulsation de résonance, $\tau$ : temps de relaxation, $\Delta M$ : intensité de relaxation, $E_{\mathrm{L}}$ : tension de ligne, $M_{\mathrm{K}}:$ masse d'un décrochement, $\varphi$ : angle moyen de la dislocation avec la direction dense, $\Phi_{\max }$ : valeur maximale de l'angle $\varphi, S$ : facteur de Schmid, $\Omega$ : facteur d'orientation ultrasonore, $b$ : vecteur de Burgers, $a$ : paramètre cristallin, $\Lambda$ : densité de dislocation, $L:$ longueur libre des dislocations, $B$ : constante de frottement, $\mu:$ module de cisaillement, $C$ : constante numérique

$$
8,68 \times 10^{-6}(\alpha \text { en } \mathrm{dB} / \mu \mathrm{s}) .
$$

Ce modèle ne peut plus s'appliquer quand la densité des décrochements géométriques (ou l'angle $\Phi$ ) croît au-delà d'une certaine valeur pour laquelle les décrochements perdent leur individualité. Si on considère que dans l'aluminium, la largeur d'un décrochement est de $10 \mathrm{~b}$ on trouve que la validité du modèle cesse quand la dislocation fait avec la direction dense un angle $\Phi$ supérieur à $\Phi_{\max }=6^{\circ}$. Au-delà la dislocation se comporte comme une corde vibrante [20]. Mais Alefeld [12] montre qu'une faible proportion de dis- locations avec $\Phi<\Phi_{\max }$ peut donner un effet de raidissement prépondérant, car l'influence des dislocations comme cordes vibrantes est très faible. On peut ainsi interpréter les résultats expérimentaux à $4 \mathrm{~K}$ puisque $p_{\alpha}$ et $p_{\mathrm{v}}$ sont proportionnels à $\Lambda L^{3}$, la figure 3 révèle la quasi-linéarité de la densité $\Lambda$ des dislocations avec la déformation plastique [21] au début de l'écrouissage. Puis l'excitation d'un second plan de glissement limite la longueur libre par enchevêtrement ce qui explique que les variations de $p_{\alpha}$ et $p_{\mathrm{v}}=f\left(\sigma_{\mathrm{e}}\right)$ présentent un palier.

En considérant : $E_{\mathrm{L}}=\mu b^{2}$,

$$
B=1,4 \times 10^{-6} \text { dyne.s. } \mathrm{cm}^{-2}\left({ }^{1}\right),
$$

$L=10^{-4} \mathrm{~cm}, \Lambda=4 \times 10^{6} \mathrm{~cm}^{-2}, \Phi=6^{\circ}$, les équations (I) donnent les valeurs suivantes

$$
\begin{aligned}
& p_{\alpha}=-40 \mathrm{mdB} \cdot \mu \mathrm{s}^{-1} / 10^{7} \mathrm{~Pa} \\
& p_{\mathrm{v}}=6 \times 10^{-11} / \mathrm{Pa}
\end{aligned}
$$

( $\left.{ }^{1}\right)$ Cette valeur est choisie plus faible que celle trouvée par Hikata et al. [23] en raison de la remarque d'Alefeld [12]. 
qui sont tout à fait compatibles avec nos mesures dans l'aluminium $6 \mathrm{~N}$ écroui à $4 \mathrm{~K}$.

La figure 4 montrant la décroissance du raidissement en fonction de la température de recuit, traduit la limitation progressive du mouvement des décrochements géométriques. En effet, lors de la déformation plastique préalable, effectuée à $4 \mathrm{~K}$, outre de nouvelles dislocations, un certain nombre de défauts ponctuels susceptibles d'interagir avec les dislocations ont été créés. Lors des recuits les mécanismes suivants peuvent être envisagés [22] :

- Réarrangement des dislocations vers les défauts ancrants, pour $T<50 \mathrm{~K}$;

- Piégeage des dislocations par les défauts du type interstitiel qui peuvent migrer vers les défauts linéaires pour $T \geqslant 50 \mathrm{~K}$.

Les variations de $p_{\alpha}$ et $p_{\mathrm{v}}$ en fonction de la température d'essai (Fig. 7) peuvent s'interpréter semiquantitativement de la manière suivante : avec $L=4 \times 10^{-4} \mathrm{~cm}, \Lambda=2 \times 10^{4} \mathrm{~cm}^{-2}$ (valeurs habituelles pour un matériau recuit) et avec les équations (I) on obtient $p_{\alpha}=20 \mathrm{mdB} \times \mu \mathrm{s}^{-1} / 10^{7} \mathrm{~Pa}$. Cette valeur correspond à la valeur moyenne de $p_{\alpha}$ observée sur la figure 7. La légère croissance de $p_{\alpha}=f(T)$ est attribuée à l'augmentation de $B$ (constante de frottement) avec la température [23].

Avec les valeurs de $L$ et $\Lambda$ précédentes, on obtient $\omega_{0} / 2 \pi=16 \mathrm{MHz}$. Dans ce cas l'équation (I) donnant $p_{\mathrm{v}}$ n'est plus valide, mais Alefeld [12] montre qu'alors $\Delta V / V$ change de sens de variation; c'est ce qui se produit autour de $80 \mathrm{~K}$ dans notre cas.

5.2 INTERPRÉTATION DE L'AMOLLISSEMENT. - Pour caractériser cet effet successif au raidissement précédemment décrit, nous nous sommes fondés sur les deux hypothèses simples suivantes :

- les variations des paramètres ultrasonores sont considérées comme la somme des variations dues au raidissement et de celles dues à l'amollissement,

- à la suite de Vincent et Perez [13], nous considérons que la variation d'atténuation due au raidissement est linéairement décroissante avec la contrainte quasi statique.

Nous pouvons ainsi décrire l'amollissement par un paramètre noté $\Delta \alpha^{*}$ tel que

$$
\Delta \alpha^{*}=\Delta \alpha\left(\sigma_{0}\right)-p_{\alpha} \sigma_{0} \text { pour } 0<\sigma_{0}<\sigma_{\mathrm{m}} .
$$

La figure 8 reporte les variations de $\Delta \alpha^{*}$ pour

$$
\sigma_{0}=250 \times 10^{-4} \mathrm{~Pa}
$$

en fonction de la température d'essai, enregistrées sur un échantillon d'Al $6 \mathrm{~N}$ déformé de $0,3 \%$ à $4 \mathrm{~K}$ et recuit $2 \mathrm{~h}$ à $220 \mathrm{~K}$. L'effet de l'amollissement apparaît ainsi principalement dans le domaine $50 \mathrm{~K}-120 \mathrm{~K}$. En rapprochant ce résultat de ceux obtenus dans un aluminium de même pureté par frottement interne et par microdéformation [17], nous interprétons ce

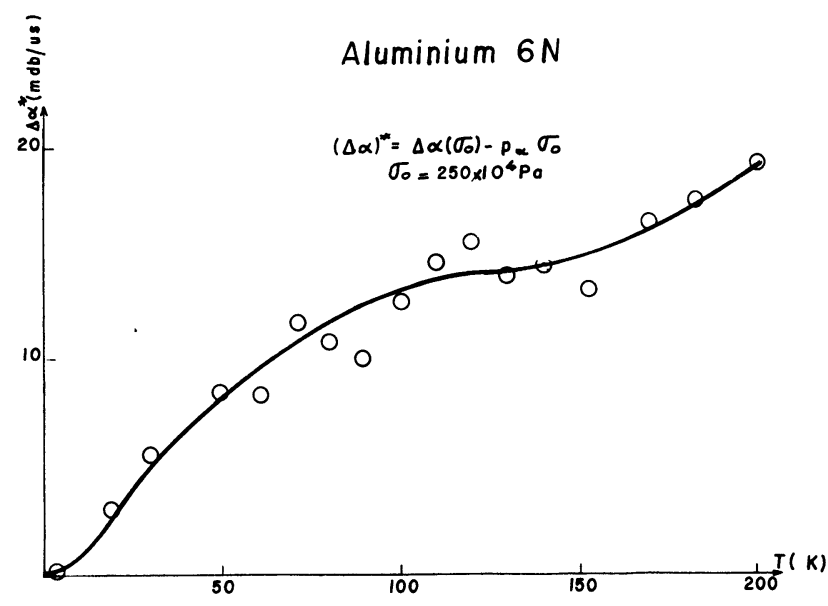

Fig. 8. - Variation de $\Delta \alpha^{*}$ pour $\sigma_{0}=250 \times 10^{-4} \mathrm{~Pa}$ en fonction de la température d'essai enregistrée dans l'aluminium $6 \mathrm{~N}$ préalablement déformé de $0,3 \%$ à $4 \mathrm{~K}$ et recuit $2 \mathrm{~h}$ à $220 \mathrm{~K}$.

[Changes of $\Delta \alpha^{*}$ for $\sigma_{0}=250 \times 10^{-4} \mathrm{~Pa}$ versus the temperature test recorded for $6 \mathrm{~N}$ aluminium cold-worked of $0.3 \%$ at $4 \mathrm{~K}$ and annealed 2 hours at $220 \mathrm{~K}$.]

phénomène par la création thermiquement activée de doubles décrochements sur les portions droites des dislocations laissées par le tassement des décrochements géométriques [24]. Des expériences récentes dans le tungstène ont conduit à des résultats similaires et une interprétation identique à la nôtre [14].

\section{3 INTERPRÉTATION DE LA MICROPLASTICITÉ. -} La courbe $\sigma_{\mathrm{m}}=f(T)$ de la figure 6 fait apparaître deux résultats :

- la limite de microplasticité décroît faiblement avec la température

- $\sigma_{\mathrm{m}}$ est bien inférieure à la contrainte de Peierls qui est vraisemblablement comprise entre $10^{-4}$ et $10^{-3} \mathrm{G}$ [25].

Dans les conditions de notre expérience, la microplasticité ne semble donc pas liée à un mécanisme de Peierls qui produirait une activation thermique beaucoup plus nette. Il semble même que cet effet masque les phénomènes dus au potentiel de Peierls puisque au-dessous de $15 \mathrm{~K}$, l'irréversibilité microplastique se déclenche avant que la création de doubles décrochements ait pu avoir lieu. Ce processus de microplasticité pourrait être dû à l'activation de sources de dislocations insensibles aux forces de Peierls (dislocations avec des angles $\Phi$ importants, dislocations à proximité de la surface... etc.). Ce type de situation, non encore explicité, a parfois été évoqué par le terme de court-circuit de déformation [25].

6. Conclusion. - Les résultats obtenus au cours de cette étude confirment le grand intérêt du couplage entre l'investigation ultrasonore et l'essai mécanique. Ces résultats, de caractère original, ne peuvent être 
expliqués dans le cadre du modèle de la corde vibrante des dislocations. Par ailleurs, le fait que les phénomènes soient beaucoup plus nets dans le cas de l'aluminium $6 \mathrm{~N}$ ne nous permet pas de retenir le rôle éventuel des atomes d'impureté. Enfin il est difficile d'envisager un effet de raidissement réversible pouvant être suivi d'un amollissement réversible à partir d'effets d'interaction entre dislocations et défauts ponctuels intrinsèques créés lors de l'écrouissage. L'hypothèse de l'existence des forces de Peierls dans l'aluminium conduit au contraire, à une description claire de nos résultats : le raidissement serait la manifestation de l'empilement des décrochements géométriques, tandis que l'amollissement serait la conséquence de la création thermiquement activée de doubles décrochements.

La confrontation entre nos résultats et ceux provenant de mesures de nature tout à fait différente (frottement intérieur et micro-déformation) a d'ailleurs permis de conforter cette hypothèse concernant les forces de Peierls [24].

Remerciements. - Nous remercions le Dr Revel qui nous a fourni l'aluminium de haute pureté.

\section{Bibliographie}

[1] Friedel, J., Riv. Nuovo Cimento 1 (1969) 110

[2] Mukherjee, A. K., Mote, J. D., Dorn, J. E., Trans. AIME 233 (1965) 1559.

[3] Guyot, P., Dorn, J. E., Can. J. Phys. 45 (1967) 983.

[4] Hikata, A., Chick, B. B., Elbaum, C., Truell, R., Acta Metall. 10 (1962) 423.

[5] VINCENT, A., Thèses Université de Lyon $(1973,1978)$

[6] Deterre, Ph., Thèse Docteur Ingénieur, Grenoble (1978).

[7] Odru, R., Riou, C., Vacher, J., Deterre, Ph., Peguin, P. et VANONI, F., Rev. Sci. Instrum. 49 (1978) 238.

[8] Cola, S., LavaitTe, A., Ros, J., VACHer, J., Note LETI/MCTE 78/188 CEN-Grenoble, 85X, 38041 Grenoble Cedex.

[9] JaOUL, R., Etude de la plasticité et applications aux métaux (Dunod, Paris) 1965.

[10] Green, R. E. et Hinton, T., Trans. AIME 236 (1966) 435

[11] Suzuki et Elbaum, J. Appl. Phys. 35 (1964) 1539.

[12] Alefeld, G., J. Appl. Phys. 36 (1965) 2642.

[13] Vincent, A., Perez, J., J. Physique-Lett. 37 (1976) 279.
[14] Pinatti, D. G. et RoberTs, J. M., 6th International Conf. of Int. Fr. and ultr. Atten. in Solids (Tokyo) 1977.

[15] Thurston, R. N. et Brugger, K., Phys. Rev. 133 (1964) 1604.

[16] Vincent, A., Perez, J., Philos. Mag. (à paraître).

[17] EsNouf, C., Thèse Lyon (1978).

[18] Vanoni, F., Thèse Grenoble (1973).

[19] Peyrade, Thèse Toulouse (1978).

[20] HiRTH, J. P. et Lothe, J., Theory of dislocations (McGraw-Hill) 1968.

[21] Keshavan, M. K. et Tillis, P. P., J. Phys. F 5 (1975) 903.

[22] Takamura, S. et OKuda, S., J. Phys. Soc. Japan 25 (1968) 714.

[23] Hikata, A., Johnson, R. A., Elbaum, C., Phys. Rev. B 2 (1970) 4856.

[24] Deterre, Ph., Esnouf, C., Fantozzi, G., Peguin, P., Perez, J., VinCent, A., VANoni, F., Acta Metall. (à paraître).

[25] Fantozzi, G., Benort, W., Esnouf, C., Perez, J., Ann. Phys. (à paraître). 